Pediatrics September 2004;114:658-662). The authors admit that sleep or sleep-deprived EEGs may provide useful information in certain epileptic syndromes (eg electrical status epilepticus during slow sleep), and the need for sleep may vary according to the clinical indications. Since these findings are contrary to general experience and opinion of many electroencephalographers, further studies are indicated.

Melatonin in wake-sleep disorders of mentally retarded children. Melatonin in bedtime doses of $3 \mathrm{mg}$ up to $9 \mathrm{mg}$ in 25 children and young adults, aged 3.6 to 26 years (mean 10.5 years), had a significant beneficial effect on sleep latency without altering the efficacy of concomitant antiepileptic drugs. (Coppola G, et al. Brain Dev Sept 2004;26:373-376).

\title{
RISK OF STATUS EPILEPTICUS IN EPILEPSY
}

The occurrence of status epilepticus (SE) after the initial diagnosis of epilepsy was determined in a prospective community-based cohort study of 613 children at Montefiore Medical Center, Bronx, NY; Yale Medical School, New Haven, CT; and BIOS/NIU, DeKalb, IL. During a median follow-up of 8 years, $58(9.5 \%)$ had 1 or $>1$ episode of SE, the first occurring a median of 2.5 years after initial diagnosis (range, $<1$ month to 8.8 years). Thirty three had only one episode, 8 had 2 episodes, and 16 had 3 or more episodes. Of 56 patients with previous SE, 18 (32.1\%) had at least one further episode during follow-up compared to 40 of $557(7.2 \%)$ without history of SE $(\mathrm{P}<0.0001)$. Factors associated with a risk of SE included SE before initial diagnosis of epilepsy, younger age at onset (3 fold increased risk at age $<1$ year vs $10+$ years) and symptomatic etiology. In those with no SE before initial diagnosis, the risk of SE during follow-up was $14 \%$ in the symptomatic etiology group vs $2.6 \%$ in the idiopathic group. In those with previous SE, the relative risks for symptomatic/idiopathic groups were $52 \%$ vs $37 \%$. Overall, $13(2.1 \%)$ died, and the risk of dying was greater in children who had previous SE (5/56 [8.9\%]) vs $8 / 557$ [1.4\%] who did not $(\mathrm{P}=0.0002)$. Death in those with previous $\mathrm{SE}$ was usually associated with an underlying cause, eg neurodegenerative or encephalopathic disorder. Children who experienced SE during follow-up were less likely to be in 3 -year remission $(19.6 \%$ vs $65.3 \% ; \mathrm{P}<0.0001)$ and much more likely to have intractable seizures $(47.4 \%$ vs $8.9 \%$; $\mathrm{P}<0.0001)$. (Berg AT, Shinnar S, Testa FM, et al. Status epilepticus after the initial diagnosis of epilepsy in children. Neurology September (2 of 2) 2004;63:1027-1034). (Reprints: Dr Anne Berg, Neuroepidemiology Group, BIOS/NIU, DeKalb, IL 60115).

COMMENT. The risk of status epilepticus (SE) in children with an initial diagnosis of epilepsy is approximately $10 \%$. The risk is increased in children with a previous history of status, in younger age groups, and in those with symptomatic etiology. Data regarding antiepileptic drug levels in patients with SE would be of interest. The authors recommend abortive therapy in the home for patients at high risk of SE. A poor outcome of SE in a Netherlands study was related to inadequate AED therapy, as well as medical complications (eg respiratory insufficiency, cardiac arrhythmias), and a duration of SE greater than 4 hours (Scholtes FB, et al. Epilepsia 1995;35:1104-1112). Noncompliance with antiepileptic drug therapy or inadequate instruction regarding the use of rectal diazepam in the home were the explanations for the admission of 8 children with SE at the University Hospital of Wales, Cardiff, UK (Matthes JWA, Wallace SJ. Dev Med Child Neurol 1995;37:226-231). 
In a previous study by the current group of authors, SE was the first seizure in $38(11 \%)$ of 342 children followed for a mean of 72 months after a first idiopathic unprovoked seizure (Shinnar S, et al. Dev Med Child Neurol 1995;37 (suppl 72):116 (abstract)). At follow-up, $127(37 \%)$ had experienced a seizure recurrence, including $42 \%$ of those who presented with status and $37 \%$ of those with a brief first seizure. SE did not adversely affect outcome in this cohort of idiopathic cases. The importance of seizure prevention or abortive therapy is stressed in both US and UK literature, especially in symptomatic epilepsies.

\section{ICTUS EMETICUS}

A 9-year-old Arabic male with a 5 year history of recurrent episodes of severe vomiting and unusual frightened behavior, was eventually diagnosed with ictal vomiting, in a report from Schneider Children's Medical Center of Israel, Petah Tiqva, and Sackler Faculty of Medicine, Tel Aviv University, Israel. Multiple interictal EEGs were all normal, but an EEG obtained at the time of an attack at age 6 years showed left frontotemporal and bilateral sharp waves followed by delta activity postictally. Symptoms during the ictal recording included vomiting, staring ahead, and chewing movements. MRI and laboratory tests for metabolic and systemic disorders were negative. Treatment with propranolol for possible migraine was successful for 7 months, and a trial of carbamazepine was effective for 1 year, followed by relapse. Topiramate was effective in controlling attacks for 1 year when vomiting recurred, and an interictal EEG showed irregular sharp and slow waves of left temporal origin and bursts of bilateral spike-wave discharges. Seizures were subsequently controlled with topiramate and valproate. The child is attending school but has attention and learning disabilities. (Shuper A, Goldberg-Stern H. Ictus emeticus (ictal vomiting). Pediatr Neurol 2004;31:283-286). (Respond: Dr Shuper, Department of Neurology, Schneider Children's Medical Center of Israel, Petah Tiqva 49202, Israel).

COMMENT. That cyclic or periodic attacks of vomiting may represent a form of epilepsy in children was proposed almost 50 years ago in a report of 33 cases studied at the Seizure Unit, Children's Medical Center, Boston (Millichap JG, Lombroso CT, Lennox WG. Pediatrics 1955;15:705-714). A history of generalized tonic-clonic or complex partial seizures in addition to vomiting attacks was found in $7(21 \%)$, and $25(76 \%)$ had seizure discharges in the interictal EEGs. Single spike discharges, usually focal and predominantly in the temporal lobe, were seen in 16 (48\%). Antiepileptic drug therapy, especially phenytoin, was partially successful in preventing vomiting attacks. Apart from one case with periodic vomiting occasionally culminating in convulsion and coma, confirmatory ictal EEGs were not obtained and, in retrospect, some of our cases may have represented attacks of atypical migraine. (see Progress in Pediatric Neurology I and III, Chicago, PNB Publ, 1991;pp4647; and 1997;51-53).

Reports of ictal vomiting recorded electrographically are scarce and most cases published have right temporal lobe involvement (Devinsky $\mathrm{O}$ et al. Neurology 1995;45:1158-1160). Kramer RE et al. (Neurology 1988;38:1048-1052) report 4 patients who underwent temporal lobectomy; and the pathology showed gliosis and neuronal heterotopia. Vomiting represented a late ictal manifestation in 3 children with occipitotemporal seizures induced by intermittent photic stimulation (Guerrini R et al. Neurology 1994;44:253-259). 